\title{
QUALITY INSPECTION FOR BLOCK ADJUSTMENT OF THE ZY3 STEREO SATELLITE IMAGERY
}

\author{
H J Tu ${ }^{1}$, Y Zhao ${ }^{1,2}{ }^{*}, \mathrm{~J} \mathrm{Guo}^{1}$, J Zhou ${ }^{1}, \mathrm{~S}$ B Dou ${ }^{3}, \mathrm{C}$ X Chen ${ }^{1}$ \\ 1 National Survey and Mapping Product Quality Inspection and Testing Center, Beijing-745783914@qq.com \\ 2 Beijing Key Laboratory of Urban Spatial Information Engineering, Beijing-704873963@qq.com \\ 3 The Third Geodetic Surveying Brigade of MNR, Chengdu-doushibiao@163.com
}

KEY WORDS: ZY3, the Block Adjustment, Quality Control, Geometric Accuracy, Accuracy, the Checking Methods.

\begin{abstract}
:
3D modeling and DEM results have played an important role in economic construction, national defense construction, social development, and ecological protection. And the regional network adjustment is the basic work of DEM, and 3D modeling. In order to ensure the quality of the following results, this paper selects the regional network adjustment results of the ZY3 stereo satellite image and puts forward the test method of the regional network adjustment results from the inspection content. Meanwhile, the northwest of Africa is selected as the test area, and the inspection contents and methods proposed in this paper are verified. The inspection results show that the quality problems existing in the adjustment results of the regional network can be effectively checked. It has a certain reference function to the quality problem of the production process.
\end{abstract}

\section{INTRODUCTION}

The regional network adjustment of satellite images is to connect several different orbital images into one area, using appropriate control points and other known data, according to the principle of least square, to solve the orientation parameters of all images and the three geographical coordinates to be fixed and to realize the accurate orientation and positioning of light at imaging time (Zhou Ping,2016). It consists of satellite image files, re-generated rational polynomial parameters (RPC) files, the regional network adjustment accuracy reports, and other satellite image supporting files, such as metadata files, browsing map files, etc.

The regional network adjustment is a key step in mapping using optical images. The quality of the adjustment results directly determines the geometric accuracy of the final product. Nowadays, 3D modeling, DEM, DSM, and other achievements have played an important role in economic construction, national defense construction, social development, and ecological protection, and so on. The regional network adjustment is the basic work of 3D modeling, DEM and DSM. It is very important to ensure the quality of the regional network At present, most of the methods of checking the accuracy of the regional network adjustment results are the method of automatically matching the same name points and manual prick points by software to check the accuracy of the results, but it is found that the accuracy quality of the regional network adjustment results is affected due to the non-standard in the production process. The adjustment results of the regional network produced in this way often appear the problem of height precision exceeding the limit when matching DSM and making DEM. In the aspect of scholars' research, Wang Mi of Wuhan University carried out an adjustment experiment on the whole network of 8802 third line array stereo pairs (26406 scene images) covering the whole country (Wang Mi,2017). The Tension of the Chinese Academy of Surveying and Mapping Sciences has proposed and developed a sparse control satellite image area network adjustment algorithm based on the general imaging model to SPOT-5 the characteristics of satellite images as CCD linear array push-scan imaging, and has carried out the regional network adjustment experiments and production in western China (Tension,2009). Zhou Ping, Wuhan University, uses SRTM to carry out elevation constraint to carry out the regional network adjustment for ZY3 satellite images (Zhou Ping,2016). Nowadays, there is no relevant technical specification for the inspection of the adjustment of the regional network of large-area satellite images, so it is necessary to form a set of inspection methods to ensure the quality of the results of the regional network. Therefore, it is very necessary to have a set of the perfect systems for quality inspection of the regional networks. In this paper, according to the characteristics of large-scale regional network adjustment results, the inspection content is formulated, the inspection method of the regional network adjustment is put forward, and the inspection method is discussed and summarized. The northwest of Africa is selected as the test area, and the inspection contents and methods proposed in this paper are verified.

\section{DATA OUTCOMES}

\subsection{The Results of the Regional Network Adjustment of Satellite Images}

Compared with aerial images, satellite images have the advantages of wide coverage, small image deformation, and low shooting cost. Therefore, in recent years, many major geographic information projects have used satellite images as base maps.

The satellite image is used to deal with the regional network adjustment, which has the characteristics of wide coverage, complex use of control data, diversification of satellite image data sources, and low overlap between models.

\footnotetext{
* Corresponding author
} 


\subsubsection{Wide Coverage}

The coverage area of single-view satellite images is generally large. For example, the coverage area of single-view satellite images is about $50 \mathrm{~km} * 50 \mathrm{~km}$. With the continuous improvement of computer processing performance, the area of a single remote sensing satellite image area network can reach 11.5 million square kilometers or even 2 million square kilometers.

\subsubsection{Complex Use of Control Data}

The use of control data will have sufficient control, rare control, or even no control because of the large area of the adjustment of satellite image area network, and it is difficult to obtain enough control points in the field completely. Even if the mode of sufficient control is used to adjust the regional network, the source of the control data is more complex. There will be a situation in which the field measurement control points, other ways to collect the field control points, high precision results data, and other control data jointly carry out the regional network adjustment.

\subsubsection{Diversification of Data Sources}

Satellite image regional network adjustment can set up different satellite platforms and different resolution satellite images in a regional network for joint adjustment. The difference of satellite image resolution in the same regional network is generally not more than 4 times.

\subsubsection{Low Overlap Between Models}

In general, the image overlap range between adjacent orbits of satellite images is not very high, so it is difficult to reach the lateral overlap degree of $60 \%$ of aerial images, and the image overlap range between adjacent orbits of the ZY3 stereo satellite images is about $10 \%$. To solve the problem of weak connection intensity between satellite image models, a processing method covering a large number of image data is generally adopted.

\section{2 the ZY3 Stereo Satellite Image Data}

the ZY3 stereo satellite is the first autonomous civilian stereo high-resolution stereo mapping satellite in China, including panchromatic image and multi-spectral image, in which panchromatic image includes three-view images, which are forward, backward, and face-up. In terms of surface pixel resolution, the front and rear visual resolution of the panchromatic image is 3.5 meters, the resolution of the face-toface image is 2.1 meters, and the resolution of the multispectral image is 5.8 meters. The width of the multispectral image is 52 $\mathrm{km}$.

\subsection{The WorldView-3 Satellite Image Data}

The WorldView-3 satellite was launched by Digital Globe in August 2014 and belongs to the fourth-generation highresolution optical satellite. At present, commercial satellites have the highest resolution, up to $0.3 \mathrm{~m}$. At the same time, the WorldView-3 satellite data in addition to the conventional red, green, blue, and near-infrared band, rare to provide 8-band data products. Because of its ultra-high resolution and multi-band data, it is widely used in various fields. The resolution of the panchromatic image is $0.31 \mathrm{~m}$, the resolution of the multispectral image is $1.24 \mathrm{~m}$, and the width of the single scene image is $13.2 \mathrm{~km}$.

\section{CONTENTS OF THE INSPECTION}

The main contents of the regional network adjustment include data quality, layout quality, and attachment quality (Zhao $\mathrm{Y}, 2020)$.

The main inspection contents of data quality include mathematical foundation, plane position accuracy, elevation accuracy, edge connection accuracy, and calculation quality. Among them, the mathematical foundation mainly includes coordinate system, elevation datum, projection parameter inspection; plane position accuracy mainly includes plane position accuracy of internal inspection point and nested accuracy of panchromatic (lower view) image and multispectral image; elevation accuracy mainly refers to elevation position accuracy check of internal inspection point; edge connection accuracy mainly includes edge connection accuracy between regional networks and edge connection accuracy check of the overlapping area of regional images; calculation quality mainly checks whether relative orientation accuracy and absolute orientation accuracy meet design requirements.

The quality of distribution points is an important part of the inspection of the regional network adjustment results. It mainly checks the quantity and distribution of control points, checkpoints, and connection points, including whether the quantity meets the requirements and whether the distribution of points is reasonable.

Attachment quality mainly checks the integrity and standardization of all kinds of documents.

Among the above inspection contents, the inspection items of plane accuracy and elevation accuracy of data quality are vital, which directly represent the geometric positioning accuracy of the regional network adjustment results. The edge-connection accuracy includes the edge-connection accuracy of the image overlapping area within the regional network and the edgeconnection accuracy between the regional networks, including the plane edge-connection accuracy and the elevation edgeconnection accuracy, which represent the internal stability of the whole regional network. Because the area of the regional network adjustment of satellite images is generally large, it even reaches 100-200 million square kilometers of each regional network, it is easy to appear the quality problems such as the stability of the regional network, which is an important inspection content of the regional network adjustment result; point quality includes the number and distribution of control points, checkpoints, connection points. control points and checkpoints have an important influence on the absolute positioning accuracy of the regional network adjustment results. the number, distribution, overlapping dimensions of the connection images play a decisive role in the relative orientation accuracy of the regional network adjustment results. Therefore, the point quality is also an important content of the quality inspection of the regional network adjustment results. The detailed inspection is shown in Table 1. 


\begin{tabular}{|c|c|c|}
\hline $\begin{array}{l}\text { Quality } \\
\text { Elements }\end{array}$ & Mass element & Check content \\
\hline \multirow{5}{*}{ Data Quality } & Mathematics Foundation & Coordinate system, elevation datum \\
\hline & Plane position accuracy & $\begin{array}{l}\text { High-precision inspection point plane accuracy compliance, panchromatic } \\
\text { image and multispectral image integration accuracy compliance }\end{array}$ \\
\hline & Elevation accuracy & Conformity of height accuracy of high precision checkpoint \\
\hline & Edge connection accuracy & $\begin{array}{l}\text { Conformity of inter-connection accuracy of block and the accuracy of edge- } \\
\text { connection of adjacent image overlapping area within block }\end{array}$ \\
\hline & Calculation quality & $\begin{array}{l}\text { Relative orientation accuracy report compliance, absolute orientation } \\
\text { accuracy report compliance }\end{array}$ \\
\hline Layout Quality & \multicolumn{2}{|c|}{$\begin{array}{l}\text { Conformity of control points, number of connection points and distribution of points, rationality of layout of } \\
\text { inspection points }\end{array}$} \\
\hline Annex Quality & \multicolumn{2}{|c|}{$\begin{array}{l}\text { 1. Completeness of submitted information } \\
\text { 2. Normative characterization of information }\end{array}$} \\
\hline
\end{tabular}

Table 1. Inspection of the results of the block adjustment

\section{INSPECTION METHODS}

Different inspection methods are adopted for different inspection contents, including internal inspection method, data audit confirmation, production data verification method, high precision data fixed point verification method, and so on. The inspection method adopts the combination of software automatic inspection and man-machine interaction (Guo $\mathrm{J}, 2020$ ).

\subsection{Data Quality}

The main inspection contents of data quality include mathematical foundation, plane position accuracy, elevation accuracy, edge connection accuracy, and calculation quality.

The mathematics foundation mainly adopts the internal inspection method, the calculation quality inspection mainly adopts the data examination confirmation, the production data verification method, the plane position precision, the elevation precision mainly adopts the high precision inspection data fixed point verification method, the panchromatic image, and the multispectral image sleeve precision, the edge connection precision inspection mainly adopts the software automatic inspection and the manual inspection method, the calculation quality mainly adopts the internal inspection method.

\subsubsection{Mathematical Basis}

Coordinate system and elevation datum can be checked by common software ArcGIS, and the basic technical indexes in the project professional technical design and implementation scheme can be compared by viewing the Spatial Reference "contents of the layer attribute" in the project.

\subsubsection{Plane Position Accuracy}

(1) For the plane position accuracy, it is detected by using the high precision checkpoint (WorldView3), and the distribution of the detection points is as uniform as possible. Because the coverage of satellite image area network adjustment results is generally large and the features of network topography are abundant, the detection points should also cover all kinds of topographic features as far as possible. The middle error does not exceed the technical design index requirement, the maximum error does not exceed twice the middle error (Wang Tao yang,2014).

(2) For the accuracy of panchromatic and multispectral images, by using software to check the matching accuracy of the panchromatic image and multispectral image, in order to ensure that the quality of the DOM results after fusion of panchromatic image and multispectral image meets the requirements, there are no quality problems such as a double image.

\subsubsection{Elevation Accuracy}

The elevation accuracy of the adjustment results of the block is detected by using the high precision checkpoint, and the elevation value is compared with the height value of the high precision checkpoint by restoring the stereo model, and the error and the maximum error are counted. In order to detect the accuracy of adjustment results (Zhang Hao,2016).

\subsubsection{Edge-connection Accuracy}

The inspection of edge connection accuracy can include the inspection of edge connection accuracy between blocks and the inspection of edge connection accuracy between models in blocks. It is also necessary to pay attention to whether the common edge area between blocks meets the technical design requirements.

(1) To check the accuracy of plane edge connection, to calculate and count the error in the edge connection and the edge connection of the panchromatic image network by software, and to check the accuracy of the elevation edge connection, mainly by using the three-dimensional model matching after adjustment DSM, to check the edge difference between the network and the network model in the weak area of the selected connection point Use the ArcGIS to find the Raster Math Minus "tool in the ArcToolbox ", subtract the DSM of the edge connection, and color the terrain section to check if there is any overrun.

(2) To check the common area range of the inter-connection edge of the block, it is mainly to use the image combination table to check whether the image range of the common edge area between the blocks is reasonable and consistent with the design. 


\subsubsection{Quality of Calculation}

The relative orientation and absolute orientation accuracy report of all points in the production process are checked by means of means of production verification. Verify that the medium error and maximum error of all accuracy reports meet the technical design requirements, focusing on the relative orientation report of connection points. Check the control point, check point adjustment report, according to different terrain categories to distinguish whether the accuracy of the inspection plane and elevation is beyond the limit, the control point residuals should be less than the requirements of the errors in the results, and the checkpoint residuals should be less than the requirements of the errors in the results. Check the residual report of the connection point, the middle error and the maximum error should meet the requirements, and the number of pixels whose error value is outside the standard of the item should not exceed $5 \%$ of the total number of connection points. Large areas of forests, deserts, waters and other difficult areas, can be appropriately relaxed index requirements.

\subsection{Layout quality}

\subsubsection{Control Points, Checkpoints}

(1) Checking the point distribution and the accuracy of the control points and checkpoints. Check whether there are systematic and regional problems in the error distribution of control points and checkpoints and check the conformity between the control points and the actual prick points of the inspection points with large error.

(2) Checking the coordinate input accuracy of control points, checkpoints. All the control points collected in the area network, checkpoints, and the actual control points used after adjustment optimization are compared in batches in the ArcGIS to confirm the accuracy of coordinate input.

(3) Checking the accuracy of the coordinate elevation system of control points and checkpoints. Because the range of adjustment results of satellite image area network is relatively large, it is difficult to measure all the control points in the field. The control points used in the adjustment process may be collected, not the latest field measurements. In order to ensure the accuracy of the elevation datum using the control points, the elevation values of the control points and checkpoints can be compared with other references to see if there is a systematic and regional error distribution. For example, if there is a systematic and regional distribution between the elevation value of the control point marked as normal height and the elevation difference of the existing normal high DEM results, and the difference value is similar to the elevation anomaly value, it can be inferred that the elevation datum of the control point may be incorrectly recorded and should be earth height.

\subsubsection{Connection Points}

The production software is used to check whether there are obvious weak areas in the connection dimension and distribution of the internal connection point and the common area connection point of the block, and to check whether there is no point in the high-dimensional overlap area of the satellite image in the weak area of the connection point distribution.

\subsection{Annex Quality}

Check whether the materials submitted are complete and complete, generally including technical design, inspection report, technical summary, etc. Whether the scope of the regional network is consistent with the technical design, and whether the actual use of the control data and the principles of use are consistent with the technical design.

\section{TEST VERIFICATION}

\subsection{Test Area}

In this paper, the northwest region of Africa is selected as the experimental area, which mainly includes the eastern part of Mali, Burkina Faso and Mauritania. Using the collected WorldView3 stereo satellite images to detect the accuracy of plane position and elevation in the test area, the distribution of WorldView3 stereo satellite images is shown in figure 1.

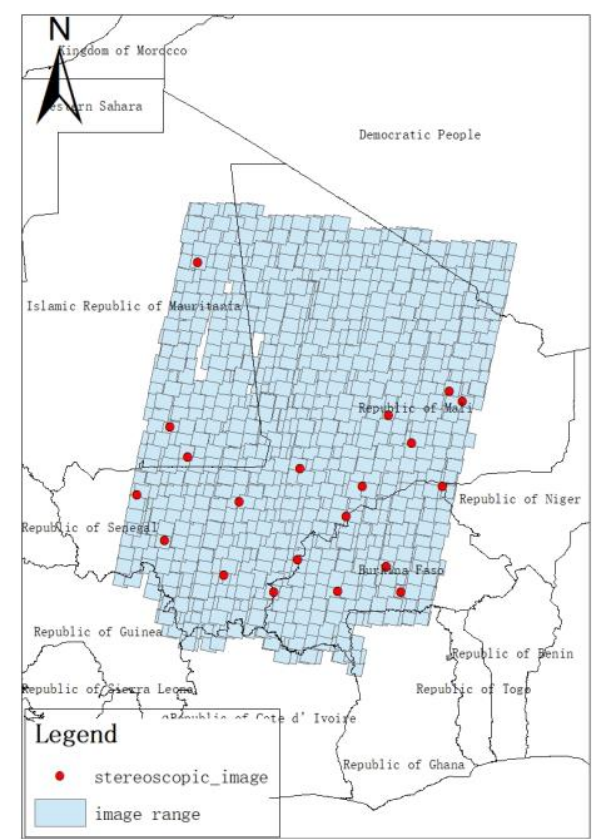

Figure 1. Image distribution of high-resolution satellites in North-West Africa

\subsection{Test Results}

After the experiment, the main problems of the regional network adjustment results are the position accuracy, the edge connection accuracy exceeding the limit, and the layout of the connection point does not meet the design requirements.

\subsubsection{Plane Position Accuracy}

(1) Plane Position Accuracy

20 plane checkpoints are selected on the collected the WorldView stereo satellite images to detect the plane position accuracy of the test area. The error statistics of the checkpoint are shown in figure 2 .

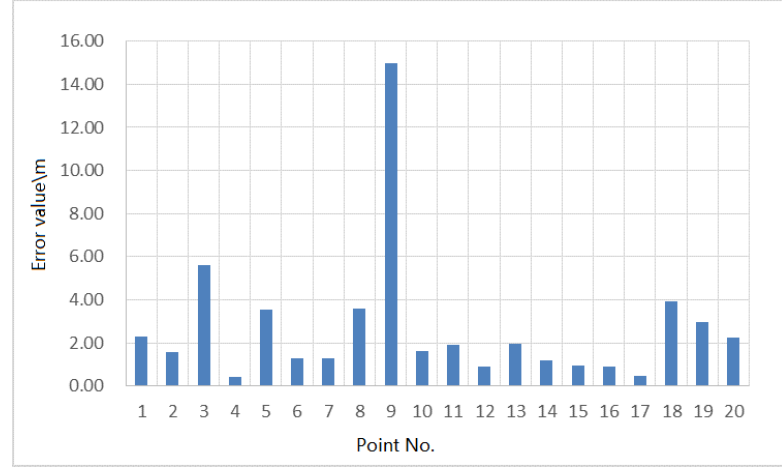

Figure 2. Distribution of plane accuracy error 
(2) Full-color and Multispectral Images

The software is used to check the accuracy of the inclusion of panchromatic image and multispectral image, and it is found that there are some problems in the inclusion of panchromatic image and multispectral image. Figure 3 6.

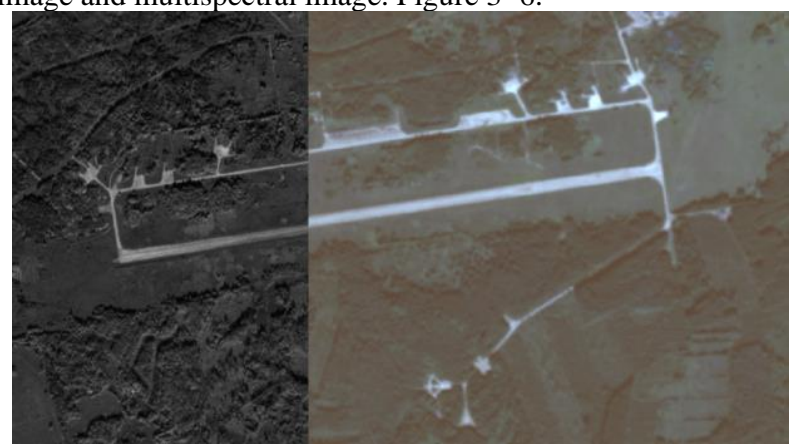

Figure 3. Full-color image and multispectral image overlay (1)

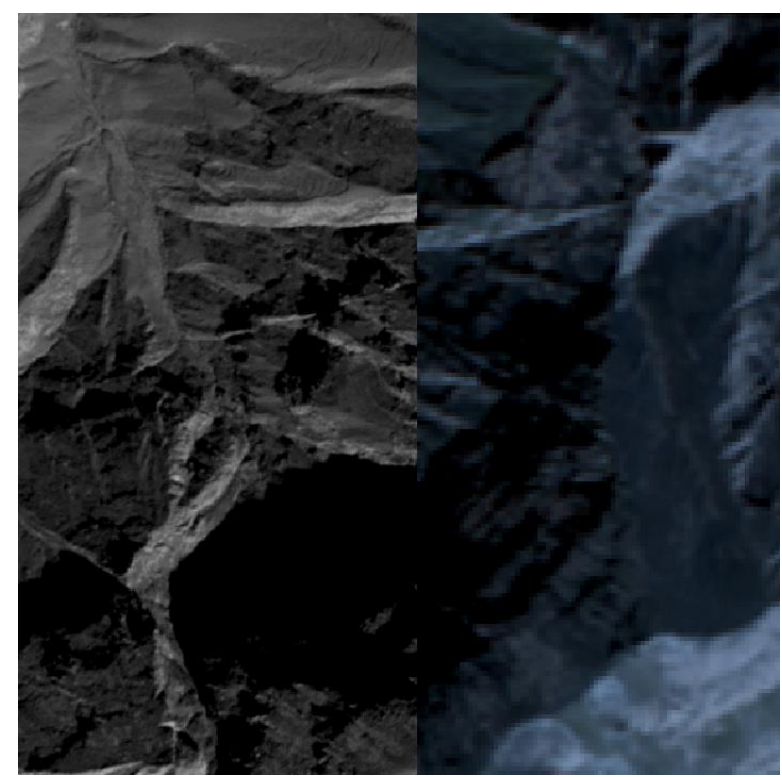

Figure 4. Full-color image and multispectral image overlay (2)

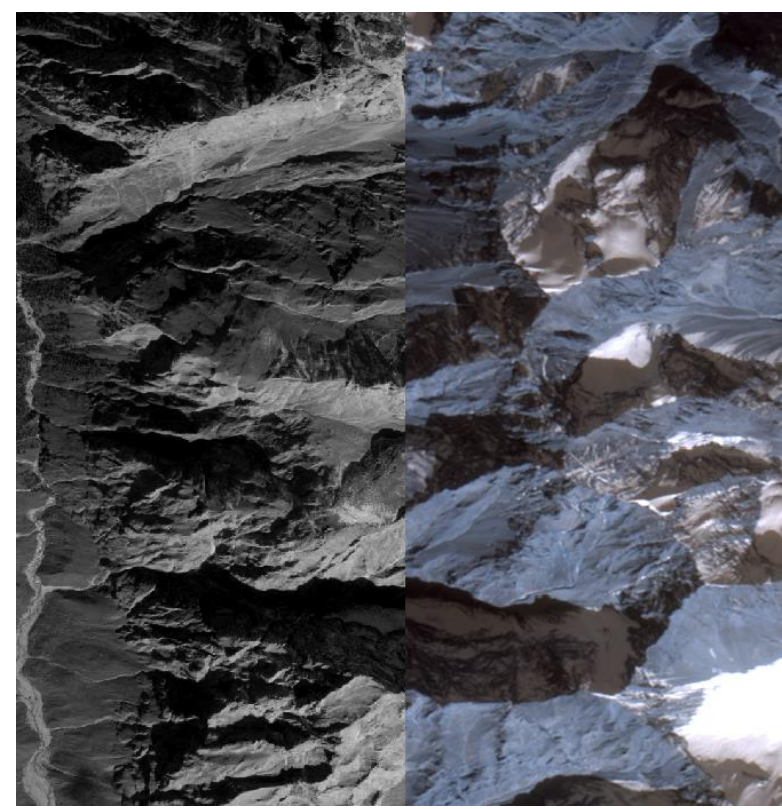

Figure 5. Full-color image and multispectral image overlay (3)

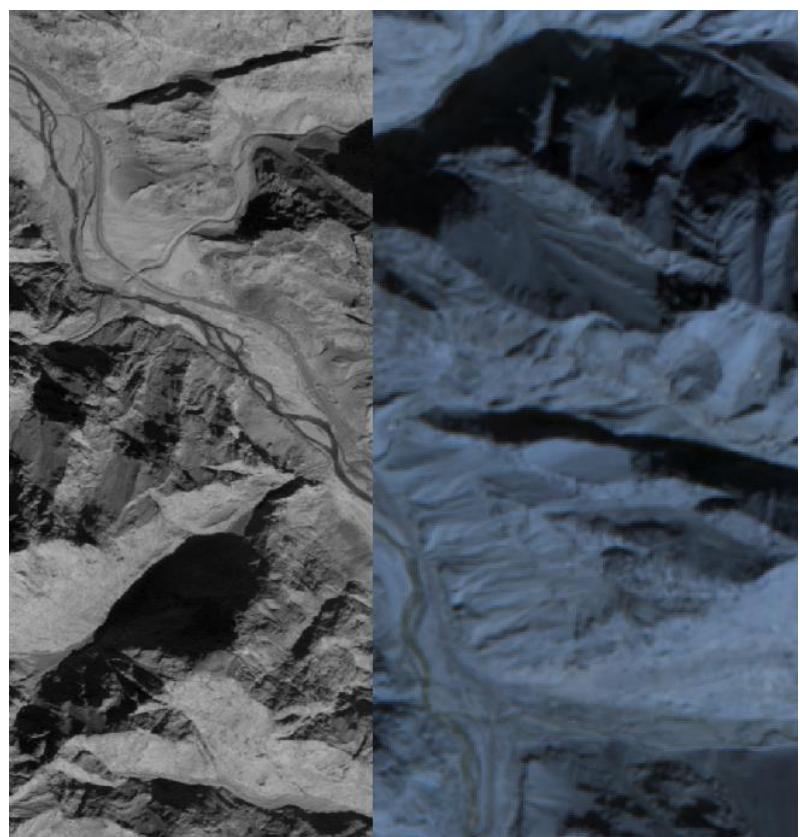

Figure 6. Full-color image and multispectral image overlay

(4)

\subsubsection{Elevation Accuracy}

20 plane checkpoints are selected on the collected the WorldView stereo satellite images to detect the accuracy of the plane position in the test area. The error statistics of the checkpoint are shown in figure 7.

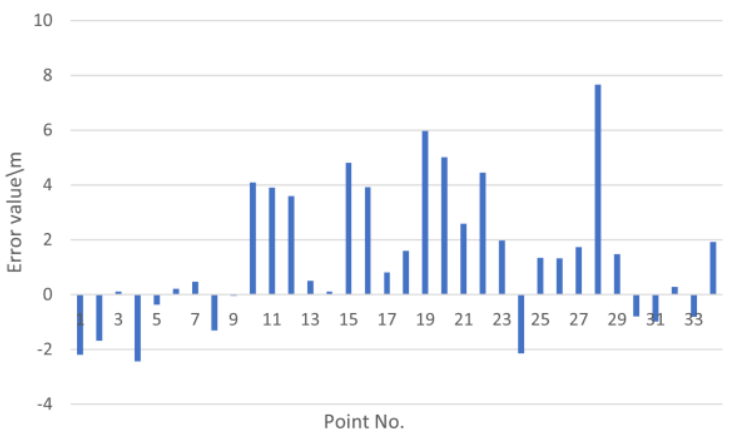

Figure 7. Error Distribution of Elevation Accuracy

\subsubsection{Edge-connection Accuracy Problem}

Using software to check the edge-connection accuracy between images in the area network, it is found that a small number of image indirect edge accuracy exceeds the limit, as shown in figure $8 \sim 12$

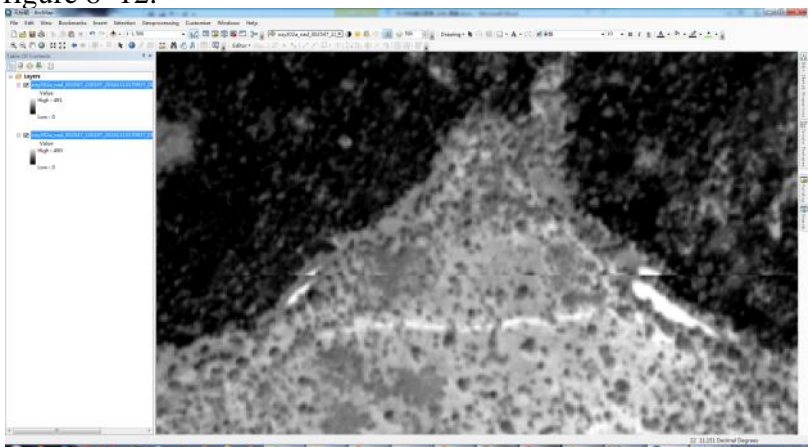

Figure 8. The accuracy of plane edge connection exceeds the limit (1) 


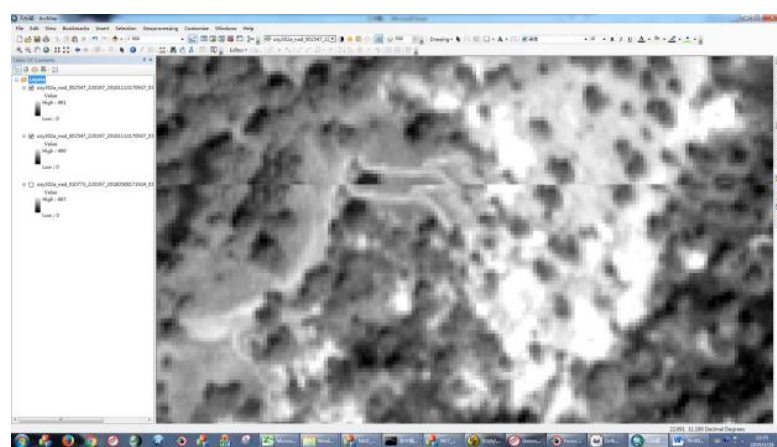

Figure 9. The accuracy of plane edge connection exceeds the limit (2)

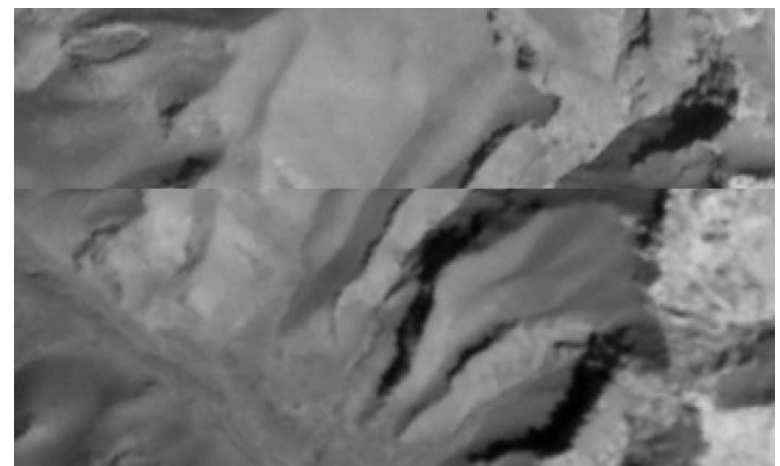

Figure 10. The accuracy of plane edge connection exceeds the limit (3)

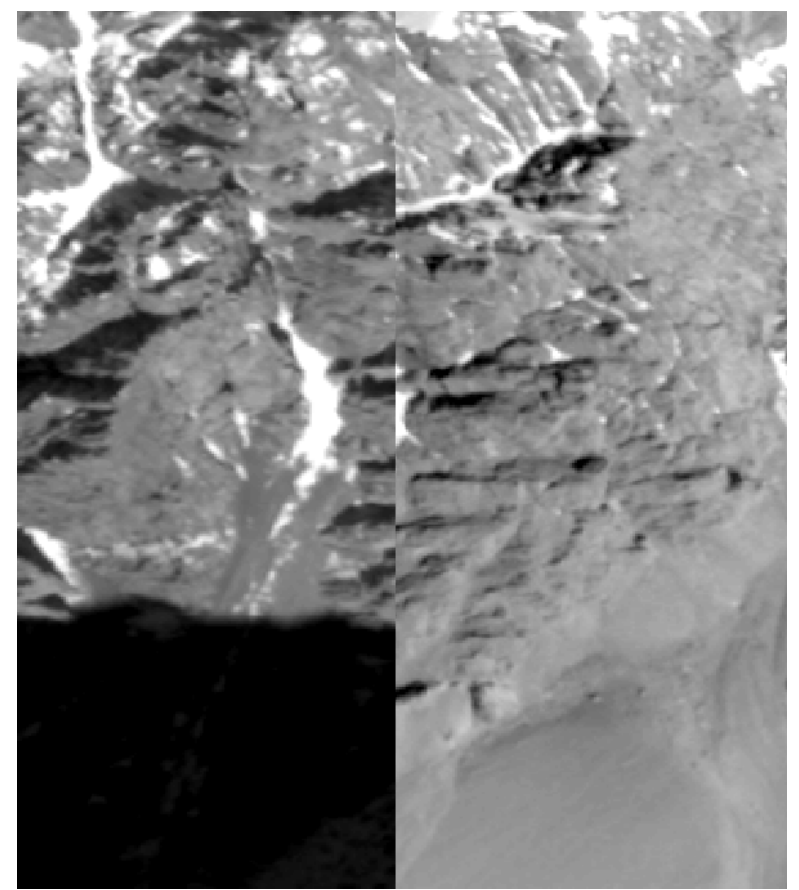

Figure 11. The accuracy of plane edge connection exceeds the limit (4)

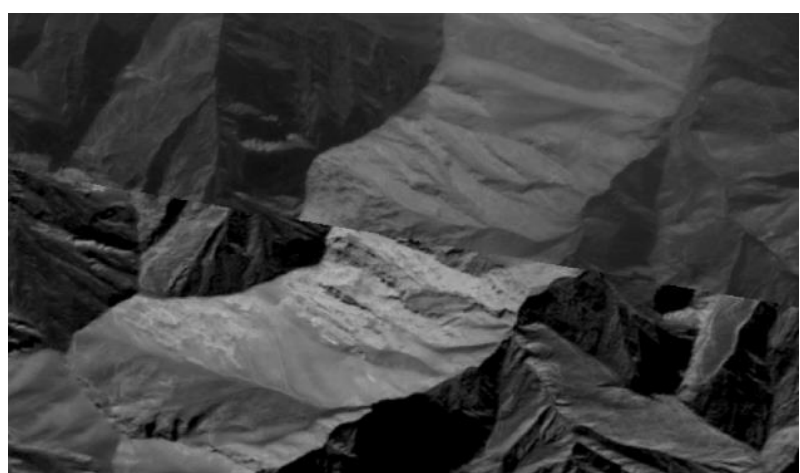

Figure 12. The accuracy of plane edge connection exceeds the limit (5)

\subsubsection{Quality of Connection Points}

By checking the number and distribution of connection points in the regional network, it is found that there are insufficient quality problems in a few areas.

As shown in figure 13 and figure 14, some regional images are more than 6 degrees overlapping, but the distribution of connection points is less.

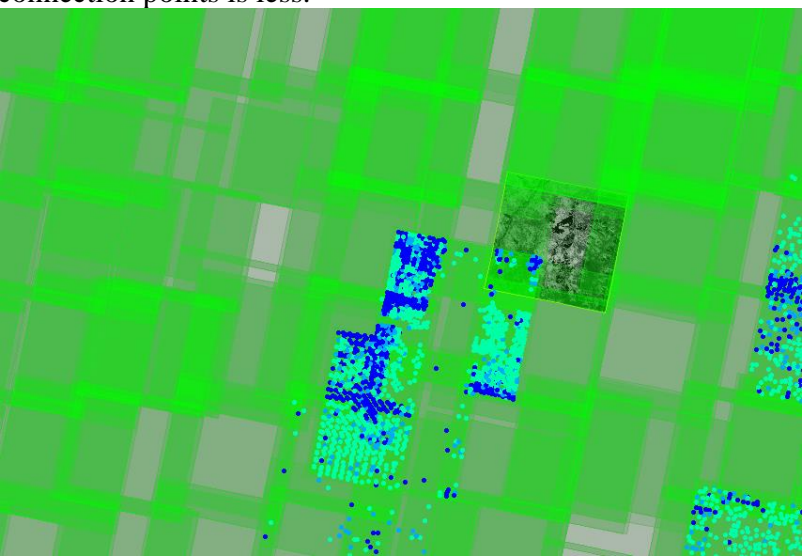

Figure 13. Unreasonable distribution of connection points (1)

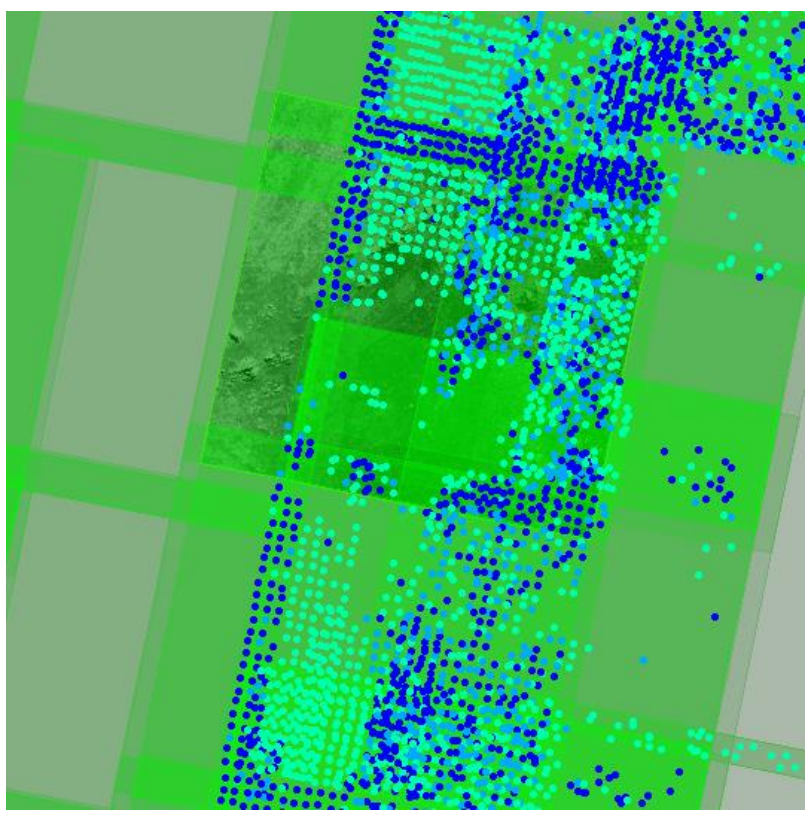

Figure 14. Unreasonable distribution of connection points (2) 


\section{CONCLUDING REMARKS}

In this paper, the method of checking the adjustment of the regional network of Resource No .3 satellite is introduced in detail and applied in the actual inspection. proves that the above inspection method is helpful to improve the efficiency of the quality inspection of the regional network adjustment.

\section{ACKNOWLEDGMENTS}

This paper is funded by Beijing Key Laboratory of Urban Spatial Information Engineering, NO. 20210223.

\section{REFERENCES}

Zhou Ping. Evaluation Method of High-precision Geometric Processing for Remote Sensing Image of ZY3 Satellite [D]. Wuhan University ,2016.

Wang Mi, Yang Bo, Li De ren, et al. Critical Technologies and Applications of Uncontrolled Whole block Adjustment in Resource No.3[J] China Journal of Wuhan University (Information Science Edition) (04): 427-433.

Tension, Zhang Ji xian, Chen Xiang yang, an Hong. Sparsely controlled SPOT-5 satellite image area network equalization [J]. Based on rational polynomial model Journal of Surveying and Mapping, v.38(04) : 302-310.,2009

Zhao Y, Zhao H T, Guo J, etal. QUALITY INSPECTION OF SATELLITE IMAGERY BLOCK ADJUSTMENT WITHOUT GCP[J]. ISPRS-International Archives of the Photogrammetry, Remote Sensing and Spatial Information Sciences,2020, XLIIIB3-2020.

Guo J, Zhang $\mathrm{J}$ X, etal. HORIZONTAL ACCURACY ASSESSMENT OF GOOGLE EARTH DATA OVER TYPICAL REGIONS OF ASIA[J]. ISPRS -International Archives of the Photogrammetry, Remote Sensing and Spatial Information Sciences,2020, XLIII-B3-2020.

Wang Tao yang, Zhang Guo, Li De ren, Jiang Wan shou, Tang Xin ming, Liu Xue lin. Comparison of the plane and threedimensional regional network adjustment of Ziyuan-3 surveying and mapping satellite image[J]. Journal of Surveying and Mapping, 2014, v.43(04):389-395 +403.

Zhang Hao, Zhang Guo, Jiang Yonghua, Wang Tao yang. Optical satellite remote sensing stereo image orthorectification controlled by SRTM-DEM[J]. Journal of Surveying and Mapping, 2016, v.45(03): 326-331+378. 